\title{
Two Distinct Nucleosome Assembly Pathways: Dependent or Independent of DNA Synthesis Promoted by Histone H3.1 and H3.3 Complexes
}

\author{
Y. Nakatani, ${ }^{*}$ D. Ray-Gallet,${ }^{\dagger}$ J.-P. Quivy ${ }^{\dagger}$ H. TAgami, ${ }^{*}$ And G. Almouzni ${ }^{\dagger}$ \\ "Dana-Farber Cancer Institute and Harvard Medical School, Boston, Massachusetts 02115; \\ ${ }^{\top}$ Section de Recherche, Institut Curie, UMR 218 du CNRS, 75248 Paris Cedex 05, France
}

Covalent modifications of histones, such as acetylation, phosphorylation, and methylation, have been shown to contribute to the formation and maintenance of transcriptionally active and inactive chromatin (Jenuwein and Allis 2001; Grewal and Elgin 2002; Turner 2002; Felsenfeld and Groudine 2003; Kurdistani and Grunstein 2003). In addition to histone modifications, histone variants that mark specific chromatin loci could play important roles in formation and maintenance of epigenetic memory (Henikoff et al. 2004). In mammals, three isotypes of histone $\mathrm{H} 3$ (H3.1, H3.2, and H3.3) have been identified in addition to the centromere-specific histone $\mathrm{H} 3$ variant, CENP-A. Histone H3.1 is the major histone H3, which is predominantly synthesized and assembled in nucleosomes during S phase. Histone H3.2 is closely related to H3.1 and has been shown to belong to the family of Sphase subtypes (Franklin and Zweidler 1977). In contrast to H3.1 and H3.2, H3.3 is expressed in proliferating cells at all stages of the cell cycle as well as in quiescent cells (Wu et al. 1982). This latter observation is consistent with the fact that $\mathrm{H} 3.3$ can be incorporated into nucleosomes in the absence of DNA synthesis. Given these properties, $\mathrm{H} 3.3-\mathrm{H} 4$ has been suggested to replace $\mathrm{H} 3-\mathrm{H} 4$ in nucleosomes, in particular during transcriptional activation (Ahmad and Henikoff 2002; Henikoff et al. 2004). Although, we are only at an early stage in our understanding of the functional role of these histone variants, it is clear that their mode of incorporation represents a crucial event with major implications for cell fate and stability of expression programs. In this context, it is remarkable to realize that while the principles for a mechanism to ensure the faithful propagation of genetic information through DNA replication were discovered a half-century ago (Watson and Crick 1953), the molecular events leading to the maintenance and transmission of epigenetic information remain a real puzzle. Much of our knowledge concerning chromatin assembly and nucleosome formation has dealt so far mainly with bulk histones (Verreault 2000; Mello and Almouzni 2001), and the issue of how to deal with histone variants, in particular $\mathrm{H} 3$, has remained

\footnotetext{
\$Present address: Division of Biological Science, Graduate School of Science, Nagoya University, Chikusa, Nagoya, Aichi 464-8602, Japan.
}

an enigma. To gain insights into how histones H3.1 and H3.3 are assembled into nucleosomes, we decided to isolate and characterize the protein complexes containing predeposition forms of these histones. Significantly, we found that histone H3.1 associates with the DNA synthesis-dependent chaperone CAF-1, while histone H3.3 associates with the DNA synthesis-independent chaperone HIRA (Tagami et al. 2004). Consistent with these observations, the H3.1 and $\mathrm{H} 3.3$ complexes mediate nucleosome assembly in a DNA synthesis-dependent and -independent manner, respectively, in vitro. Most strikingly, our data point to the existence of histones $\mathrm{H} 3$ and $\mathrm{H} 4$ in both predeposition complexes as a dimer (Tagami et al. 2004). In the present paper, we will summarize these recent findings to highlight the potential of the experimental strategies we developed. We will then discuss how we can envisage the relevance of a $\mathrm{H} 3-\mathrm{H} 4$ dimer, rather than a tetramer, as a basic "building unit" for chromatin dynamics. Finally, possible implications of our findings for mechanisms involved in nucleosome assembly and epigenetic inheritance will be considered to help define some promising new avenues and to stimulate future developments in the field.

\section{HISTONE DEPOSITION COMPLEXES}

To explore the mechanisms of how histones H3.1 and H3.3 are assembled into chromatin, we purified preassembled histones H3.1 and H3.3 from the nuclear extract fraction of HeLa cells expressing epitope-tagged H3.1 or H3.3, respectively (Tagami et al. 2004). For immuno-affinity purification (Nakatani and Ogryzko 2003), we have fused the FLAG (DYKDDDDK) and HA (YPYDVPDYA) epitone sequences to the carboxy-terminal end of histones H3.1 (e-H3.1) or H3.3 (e-H3.3). To optimize accessibility to the epitope tag, and to avoid major interference with normal functions of histones $\mathrm{H} 3.1$ and H3.3, we inserted a "flexible" linker, AAAGG, between the carboxy-terminal end of histones H3.1 or H3.3 and the epitope tag. Immunofluorescent staining of the mitotic cells expressing e-H3.1 or e-H3.3 reveals that most of the tagged histones colocalize with chromosomes, indicating that the epitope-tagged $\mathrm{H} 3.1$ and $\mathrm{H} 3.3$ could be effectively assembled into nucleosomes in vivo. 
a

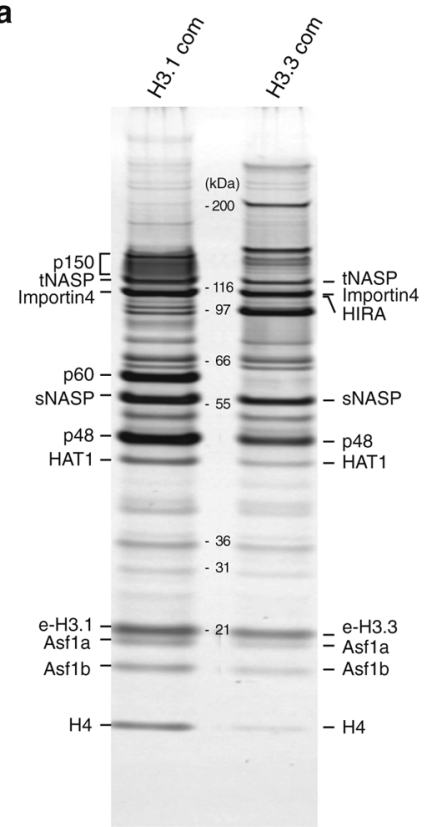

b
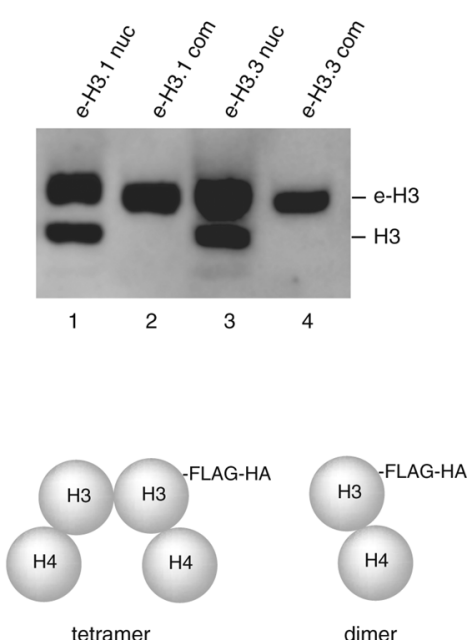

dimer

Figure 1. (a) Compositions of the H3.1 and H3.3 complexes. Silver staining of the H3.1 and H3.3 complexes (H3.1 com and H3.3 com). The polypeptides identified by mass spectrometric analyses are indicated. (b) Histones $\mathrm{H} 3$ and $\mathrm{H} 4$ in the $\mathrm{H} 3.1$ and $\mathrm{H} 3.3$ complexes exist as a dimer. (Top) Immunoblotting of mononucleosomes and nucleosome assembly complexes containing epitope-tagged histone H3.1 and H3.3. e-H3.1-containing (lane 1) and e-H3.3-containing (lane 3) mononucleosomes and the H3.1 (lane 2) and H3.3 (lane 4) complexes were analyzed by immunoblotting with anti-histone $\mathrm{H} 3$ antibody. The positions for epitope-tagged histone $\mathrm{H} 3$ (e$\mathrm{H} 3.1$ or e-H3.3) and native $\mathrm{H} 3$ are indicated. (Bottom) Given that untagged H3 is far more abundant than epitope-tagged H3 in the cell lines employed, the epitope-tagged H3 could preferentially pair with untagged H3 to form the H3-H4 tetramer. Accordingly, mononucleosomes contain epitope-tagged and untagged $\mathrm{H} 3$ (lanes 1 and 3 ). In contrast, the H3.1 and H3.3 complexes have no detectable untagged $\mathrm{H} 3$, indicating that histones $\mathrm{H} 3$ and $\mathrm{H} 4$ in these complexes exist as a dimer. (Adapted, with permission, from Tagami et al. 2004 [@Cell Press].)

The H3.1 complex resolution is shown in Figure 1a as revealed after analysis by PAGE and silver staining. Table 1 summarizes the subunit composition of the different complexes that we characterized, with a list of the polypeptides identified so far. The H3.1 complex con- tains two distinct histone chaperones for $\mathrm{H} 3-\mathrm{H} 4$; the entire set of CAF-1 subunits (p150, p60, and p48) (Smith and Stillman 1989) and the two isoforms of Asf1 that exist in human cells (Asfla and b) (Munakata et al. 2000; Sillje and Nigg 2001; Tyler et al. 2001; Mello et al. 2002).

Table 1. Nucleosomal and Nonnucleosomal Complexes Containing Epitope-Tagged H3

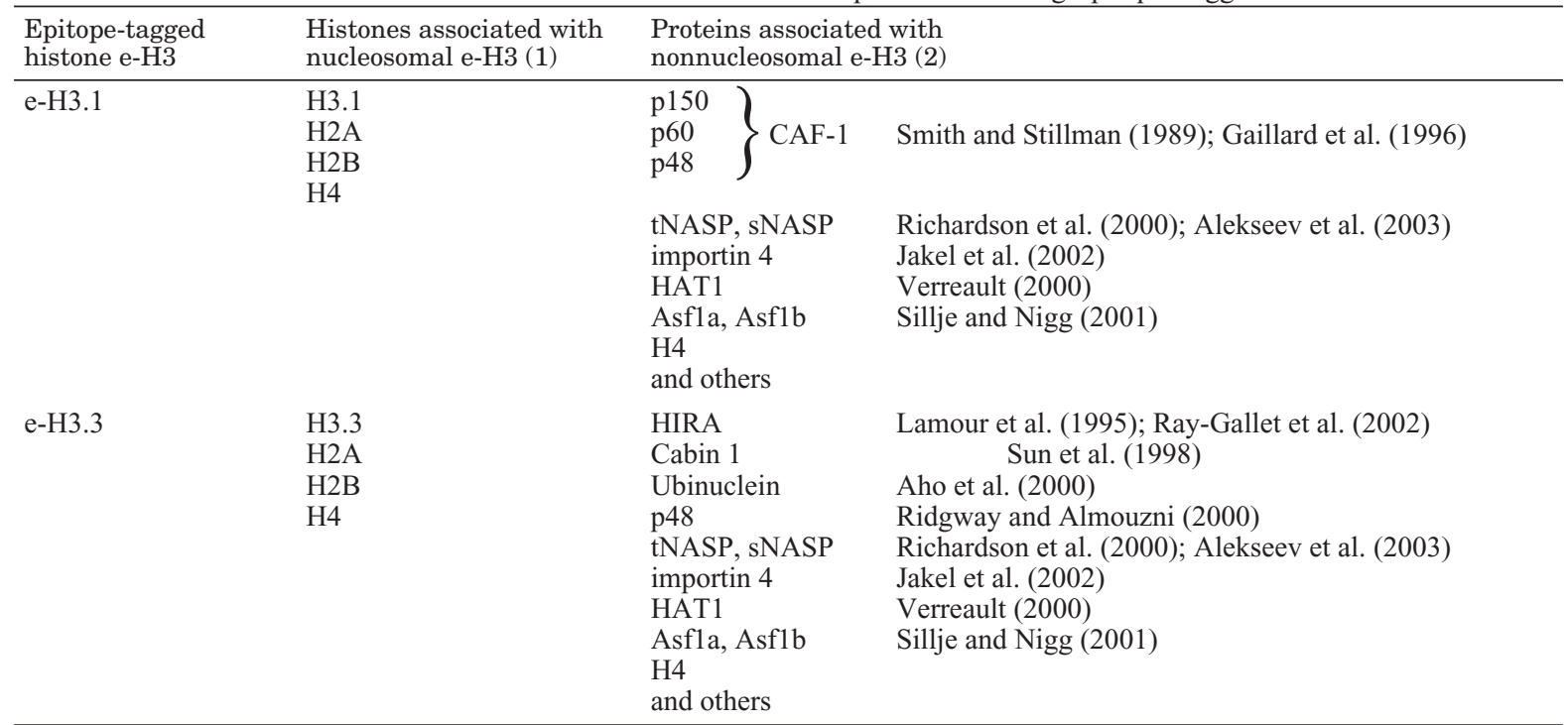

\footnotetext{
Affinity purification was carried out using (1) mononucleosomes isolated after glycerol fractionation of MNase digestion products, and (2) nuclear extracts prepared according to Dignam et al. (1983).
} 
Moreover, the complex contains NASP, which is a candidate for histone H1 chaperone (Richardson et al. 2000; Alekseev et al. 2003). In addition to these histone-binding proteins, the complex contains the histone-acetylating enzyme HAT1, which can modify predeposited histones H3 and H4 (Verreault 2000), as well as importin 4, which presumably mediates nuclear translocation (Jakel et al. 2002). Glycerol gradient sedimentation of the purified complex suggests that most histone $\mathrm{H} 3.1$ belongs to a major complex.

We also purified preassembled histone H3.3 from the nuclear extract and the subunit composition of the H3.3 complex is shown in Figure 1a and Table 1. Glycerol gradient sedimentation profiles of the purified complex show that H3.3 belongs to at least three subcomplexes. Importantly, among these three complexes, one of them contains HIRA, a DNA synthesis-independent histone chaperone (Lamour et al. 1995; Ray-Gallet et al. 2002). This important feature was a first demonstration of a link between histone H3.3 and HIRA. Western blotting analysis confirmed that HIRA is specific to the H3.3 complex, while CAF-1 is specific to the H3.1 complex. As described later, this difference could explain why H3.1 and H3.3 are deposited into nucleosomes through distinct pathways. Moreover, Cabin 1 (Sun et al. 1998) and ubinuclein (Aho et al. 2000) are found in the HIRA-containing complex. Subunits in common with the H3.1 complex were Asf1, importin 4, HAT1, and p48 (Ridgway and Almouzni 2000), which suggests that they could play related roles in both complexes.

\section{HISTONE H3-H4 EXISTS AS A DIMER IN THE NUCLEOSOME ASSEMBLY COMPLEXES}

Although histones $\mathrm{H} 3$ and $\mathrm{H} 4$ are known to form tetramers under physiological conditions, several lines of evidence support the fact that the dimeric form of histones $\mathrm{H} 3$ and $\mathrm{H} 4$ could exist within predeposition complexes associated with chaperones. Western blotting analysis of the H3.1 and H3.3 complexes with anti-histone H3 antibody shows that these complexes contain epitopetagged histone H3 (e-H3.1 or e-H3.3), but not untagged H3 (Fig. 1b, lanes 2 and 4). Yet, we should consider that the epitope-tagged histones are far less abundant than the endogenous histones in our cell lines. Thus, as illustrated in Figure $1 \mathrm{~b}$, if histone $\mathrm{H} 3-\mathrm{H} 4$ were to exist as a tetramer in a predeposition form, statistically one would expect the complex to contain both epitope-tagged and untagged $\mathrm{H} 3$, which is not the case. In contrast, mononucleosome core particles purified by anti-FLAG antibody immunoprecipitation contain both epitope-tagged and untagged H3 (Fig. 1b, lanes 1 and 3). These data lead us to conclude that histones $\mathrm{H} 3$ and $\mathrm{H} 4$ in the predepostion nucleosome assembly complexes are present as dimers, rather than tetramers. The existence of $\mathrm{H} 3-\mathrm{H} 4$ dimers as intermediates in chromatin dynamics will be discussed later. It is also important to note that, within the mixed population as tagged and untagged versions of histone $\mathrm{H} 3$ in our mononucleosomes, the variants involved were identical, as shown by the mass spectrometry analysis. It is still unclear how some kind of "recognition" can bring together the same kind of variants within the same particle. However, this can provide interesting information concerning how the differences in sequences between H3.1 and H3.3 may have implications for their association as tetramers.

\section{HIRA- AND CAF-1-DEPENDENT ASSEMBLY PATHWAYS}

The H3.1 and H3.3 complexes isolated from nuclear extracts as shown above contain distinct histone chaperones. Most remarkably, all three subunits of CAF-1p150, p60, and p48 - are associated with H3.1, consistent with deposition coupled to DNA replication as expected, while HIRA is associated with the H3.3 complex (Fig. 1 and Table 1). Indeed, these two chaperones have been shown to promote distinct nucleosome assembly pathways in higher eukaryotes. HIRA is critical for a DNA synthesis-independent nucleosome assembly process (Ray-Gallet et al. 2002), while CAF-1 mediates nucleosome formation coupled to DNA synthesis during DNA replication (Smith and Stillman 1989) or DNA repair (Gaillard et al. 1996). To test the capacity of our complexes in each of these assembly pathways, the potent nucleosome assembly capacity of Xenopus egg extract system (HSE) is a convenient system (Ray-Gallet and Almouzni 2004). Pioneering work using Xenopus egg extracts (Laskey et al. 1977) provided the basis for analyzing different assembly pathways by immunodepleting HIRA or p150 CAF-1 from HSE, as shown in Figure 2a.

HIRA-depleted HSE supports only nucleosome assembly coupled to DNA synthesis using UV-treated plasmid DNA (DNA UV), to follow nucleosome assembly in the presence of DNA synthesis during nucleotide excision repair, whereas CAF-1-depleted HSE supports only nucleosome formation in the absence of DNA synthesis using intact plasmid DNA (DNA 0) (Fig. 2b). With such a system in hand, we could test our different purified H3.1 and H3.3 complexes (and subcomplexes) for their ability to rescue the defect in nucleosome assembly of the depleted extracts. Results from these data showed that the histone H3.1 and H3.3 complexes mediate DNA synthesis-dependent and DNA synthesis-independent nucleosome assembly, respectively. Although the specificity of each pathway could be dictated by the two chaperones - CAF1 (for H3.1) and HIRA (for H3.3), respectively (Loyola and Almouzni 2004) - the basis of the specific interaction of the distinct deposition complexes with H3.1 and H3.3 is still unclear. Given that specific interactions of HIRA with H3.3 and CAF-1 with H3.1 could not be revealed in assays using recombinant proteins (not shown), formation of specific complexes is likely to involve additional aspects, in vivo, which will have to be understood in future studies. Furthermore, CAF-1 and HIRA are not necessarily found only in histone-containing complexes. This has been recently illustrated by the fact that CAF-1 was found in a complex containing HP1 $\alpha$, which was devoid of histone H3 (Quivy et al. 2004). In addition, the function of HIRA has been associated with repression of histone transcription (Nelson et al. 2002), as found initially in Saccharomyces cerevisiae for Hir1 and Hir2 (Sherwood et al. 1993). Whether this is related to the function of the com- 
a

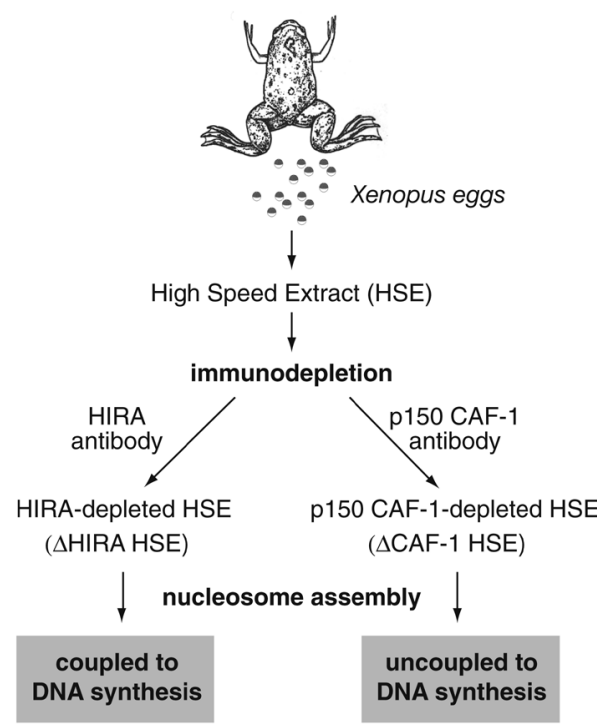

b
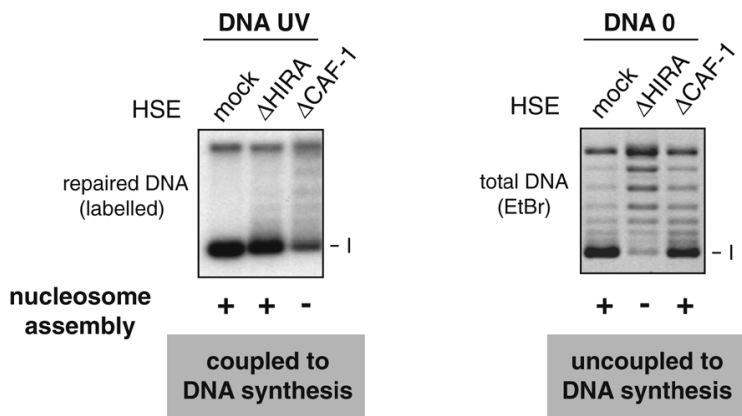

Figure 2. Two distinct chromatin assembly pathways: one coupled to DNA synthesis and CAF-1 mediated, and another uncoupled to DNA synthesis and HIRA dependent. (a) Immunodepletion strategy used to monitor these two distinct chromatin pathways individually. Xenopus egg extract (HSE), supporting both pathways, is depleted either of HIRA or of the p150 subunit of CAF-1 by using specific antibodies. HIRA-depleted HSE ( $\triangle$ HIRA HSE) supports only chromatin assembly coupled to DNA synthesis, whereas p150 CAF-1-depleted HSE ( $\triangle \mathrm{CAF}-1 \mathrm{HSE}$ ) supports only chromatin assembly uncoupled to DNA synthesis. (b) Analysis of the nucleosome assembly activities coupled and uncoupled to DNA synthesis in $\triangle$ HIRA and $\triangle \mathrm{CAF}-1$ HSEs. Mock, $\triangle \mathrm{HIRA}$, or $\triangle \mathrm{CAF}-1$ HSEs were used in nucleosome assembly assays that were carried with either UV$\mathrm{C}$-treated plasmid (DNA UV) in the presence of $\alpha_{-}{ }^{32} \mathrm{P}(\mathrm{dCTP})$ to follow the nucleosome assembly pathway coupled to DNA synthesis or nonirradiated plasmid (DNA 0 ) to follow the DNA synthesis-independent pathway. The repaired and nonrepaired assembled DNA were analyzed by supercoiling assay and visualized by autoradiography and by ethidium bromide staining, respectively. Migration position of DNA plasmid form I (supercoiled) corresponding to the assembled DNA is indicated. (Adapted, with permission, from Ray-Gallet and Almouzni 2004 [@Elsevier].)

plex that we have studied here or to another one remains to be elucidated. Thus, the functions of CAF-1 and HIRA in histone deposition are thus likely to be important in multiple contexts. In any case, distinct pathways can be promoted by the distinct histone-containing complexes in which we found, respectively, CAF-1 and HIRA.

\section{HISTONES H3.1 AND H3.3 ARE DEPOSITED THROUGH DISTINCT NUCLEOSOMAL ASSEMBLY PATHWAYS}

Having shown that nuclear complexes containing histones H3.1 and H3.3 could promote distinct nucleosomal assembly pathways, it was important to determine if histones H3.1 and H3.3 are indeed specifically deposited onto DNA through such distinct pathways. Cytosolic extracts from HeLa cells expressing e-H3.1 or e-H3.3, which contain no detectable CAF-1 or HIRA, provided us with a source of free-tagged histones for nucleosomal assembly reactions. We then used a DNA linked to bead assay, as shown in Figure 3a, to analyze histone deposition "de novo," i.e., to test nucleosome formation on a template free of histone. To examine DNA synthesis-dependent nucleosomal assembly, UV-damaged DNA immobilized onto magnetic beads was incubated with HIRA-depleted HSE in the presence of cytosolic extracts containing e-H3.1 or e-H3.3. In these HIRA-depleted extracts, which are still functional for the CAF-1-dependent assembly pathway, histones bound to immobilized DNA could be analyzed by immunoblotting. e-H3.1 was indeed recovered onto immobilized UV-damaged DNA in a CAF-1 (from the HSE)-dependent manner (Fig. 3b, left, lanes 2 and 4). In contrast, no e-H3.3 was detected on the immobilized DNA (Fig. 3b, left, lane 8). Thus, histone H3.1, but not H3.3, is utilized by the DNA synthesis-dependent nucleosome assembly pathway.

In parallel, we also examined the deposition of histones H3.1 and H3.3 in a DNA synthesis-independent nucleosomal assembly pathway. This time, we used immobilized intact DNA as a template and a CAF-1 p150-depleted HSE in which only the HIRA-dependent pathway is functional. In contrast to the DNA synthesis-dependent deposition, e-H3.3, but not e-H3.1, was recovered onto immobilized intact DNA (Fig. 3b, right, lanes 4 and 8), indicating that histone H3.3 is specifically deposited in DNA synthesis-independent nucleosome assembly.

We conclude that in this system it is possible to reveal a specific loading of histone $\mathrm{H} 3.1$ and $\mathrm{H} 3.3$ onto DNA that occurs using DNA synthesis-dependent and -independent "de novo" nucleosome assembly pathways, respectively (Fig. 3c). In such a scheme one would predict H3.1 loading during DNA replication in S phase or during repair synthesis. H3.3 loading would occur throughout the cell cycle. Given that we have not been able to detect a loading of H3.3 coupled to DNA synthesis in vitro, a postreplicative mode of deposition could perhaps be considered for $\mathrm{H} 3.3$ during $\mathrm{S}$ phase.

\section{"DE NOVO" DEPOSITION THROUGH TWO DISTINCT PATHWAYS USING DIMERS}

Importantly, the purification scheme of the distinct H3 (H3.1 or H3.3) complexes suggested that they were present in dimeric form together with histone $\mathrm{H} 4$ in the nuclear extract. A simple way to explain the mixed population of $\mathrm{H} 3$ (tagged and nontagged) produced in mononucleosomes isolated from our cells could be according to the model in Figure 4 with two nonexclusive options. In the first case, two dimers of one type are 
a
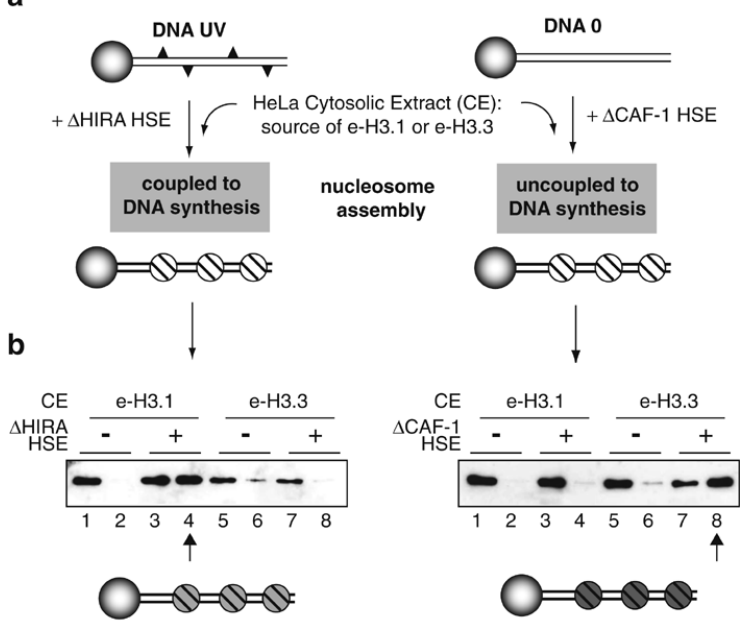

H3.1 containing

nucleosomes

C
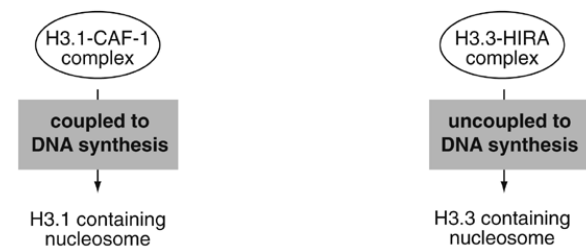

Figure 3. Histone H3.1 is specifically deposited via a nucleosome assembly pathway coupled to DNA synthesis, whereas histone H3.3 is specifically deposited via a nucleosome assembly pathway independent of DNA synthesis. (a) Scheme of the experiment: Magnetic bead-linked UV-irradiated DNA (DNA UV) or intact DNA (DNA 0) were incubated with cytosolic extracts from HeLa cells expressing either e-H3.1 or e-H3.3 complemented with $\triangle$ HIRA HSE for the UV-irradiated DNA or $\triangle \mathrm{CAF}-1 \mathrm{HSE}$ for the intact DNA. The nucleosome assembly pathway coupled to DNA synthesis supported by the $\triangle$ HIRA HSE extract occurs during the DNA repair synthesis of the UVirradiated DNA. The nucleosome assembly pathway uncoupled to DNA synthesis supported by the $\triangle \mathrm{CAF}-1$ HSE occurs on the intact DNA. (b) Analysis by Western blot of the histones H3.1 and $\mathrm{H} 3.3$ deposition during the nucleosome assembly pathways either coupled or uncoupled to DNA synthesis. Bead-DNA was incubated with cytosolic extract containing e-H3.1 (lanes 1-4) or e-H3.3 (lanes 5-8) in the absence (lanes 1,2, 5, and 6) or presence (lanes 3, 4, 7, and 8) of immunodepleted HSE. Histones $\mathrm{H} 3.1$ and H3.3 were detected by anti-HA antibodies as $10 \%$ input of the reaction (odd lanes) and bound material (even lanes). (c) Hypothetical nucleosome assembly line where the H3.1-H4 dimer specifically associated with CAF-1 helps to form H3.1containing nucleosome by a DNA synthesis-dependent nucleosome assembly pathway. The H3.3-H4 dimer specifically associated with HIRA helps to form H3.3-containing nucleosome by a DNA synthesis-independent nucleosome assembly pathway. (Adapted, with permission, from Tagami et al. 2004 [@Cell Press].)

loaded in a coordinated manner by two identical chaperone complexes that play an equivalent role in the specific loading reaction (Fig. 4a). Alternatively, one dimer could be targeted onto DNA through the specific dedicated chaperone (complex A) and another complex (complex B) would provide the second dimer (the origin of which is discussed later) (Fig. 4b). In the latter scenario the specificity for one pathway or another would be dictated by a component in complex A, such as CAF-1 for a pathway coupled to DNA synthesis to promote H3.1 loading, or HIRA for the pathway independent of DNA synthesis to promote H3.3 deposition. Obviously some cross talk between complexes A and B would be needed, which could perhaps be achieved by a shuttling between the complexes of certain subunits. An attractive candidate for such a histone-shuttling activity could be Asf1. Present in both cytosolic and nuclear fractions in HeLa cells (Mello et al. 2002), Asf1 could potentially act both as a histone acceptor, as recently proposed in yeast (Adkins et al. 2004), and as histone donor in an assembly line (Koundrioukoff et al. 2004; Loyola and Almouzni 2004). This would be consistent with the fact that both the H3.1 and H3.3 complexes possess this additional histone H3-H4 chaperone, Asf1 (Fig. 1 and Table 1). Given that both CAF-1 and Asfl have been independently shown to interact with $\mathrm{H} 3-\mathrm{H} 4$, an additional $\mathrm{H} 3-\mathrm{H} 4$ dimer could potentially be used to provide the two $\mathrm{H} 3-\mathrm{H} 4$ dimers necessary to form a tetramer. A similar mechanism could be at work with the H3.3 complex.

Thus, a "de novo" assembly pathway as followed in our in vitro system using naked DNA as a substrate may also be used in vivo in situations where DNA becomes available in a "histone-free" form, subsequent to some disruptive event, such as is found during DNA replication, transcription, repair, or recombination.

\section{H3-H4 DIMERS AS INTERMEDIATES DURING CELLULAR LIFE: HISTONE FATE AND NUCLEOSOME DYNAMICS}

Our data collectively point to the existence of $\mathrm{H} 3-\mathrm{H} 4$ dimers as intermediates that have to be considered during cellular life. In principle, dimeric forms of H3-H4 could originate from various possible sources, as summarized in a

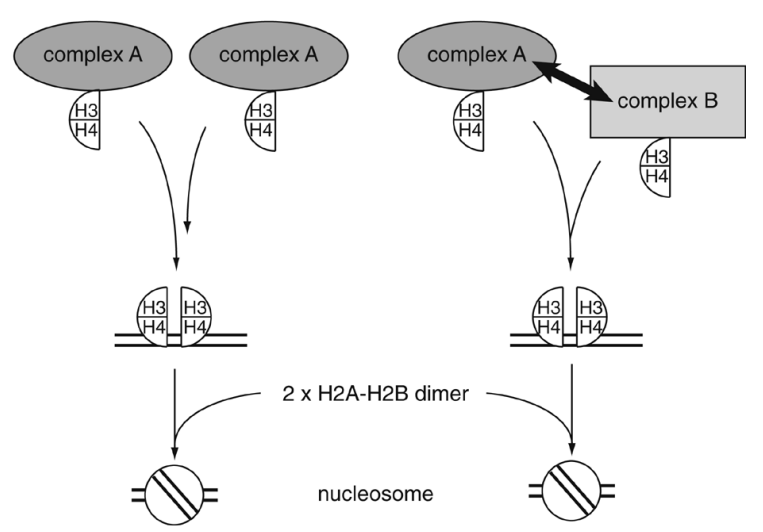

Figure 4. Hypothetical model for nucleosome assembly from H3-H4 dimer-containing complexes. (a) Two H3-H4 dimers are deposited onto DNA by two identical complexes (A) in one step or in two subsequent steps. Following association of two H2A-H2B dimers, a nucleosome is formed. (b) Two distinct H3-H4-containing complexes (A and B) are used. 
our scheme in Figure 5. The first option could be that all dimers originate from newly synthesized histones that are to be incorporated into nucleosomes "de novo" (Fig. 5, left panel). In such a case, it will be particularly important to examine which modifications are associated with the histone-containing complexes and when they are imposed. The relative enrichment in chromatin of specific combination of histone modifications associated with each of the variants to distinguish active from silent chromatin found in Alfafa and Drosophila cells (Waterborg 1990; McKittrick et al. 2004) could be strictly dependent on the nature of the variants and the corresponding specific complexes. In this way specific patterns could be dictated at rather early stages soon after synthesis. Although this is a simple solution at first glance, it remains unclear how subtle distinct patterns for different types of silent regions can be established. In mammals, for example, how can the distinction be made between constitutive and facultative heterochromatin as found on the inactive $\mathrm{X}$ chromosome in females (Heard 2004; Maison and Almouzni 2004) and how can specific developmentally regulated expression be tightly regulated in a more subtle way than just on/off? Surely, in addition to a certain degree of specificity provided by histone variants and corresponding associated complexes, one will have to also consider the contribution of additional modifications targeted by other means through specific modifying enzymes.

A second potential source of $\mathrm{H} 3-\mathrm{H} 4$ dimers would be when parental histones (i.e., initially already present in a nucleosomal form) are recycled (Fig. 5, middle and right panels). At one extreme, if the recycling does not involve disruption of a tetramer, or only a transient dissociation followed by rapid reassociation, no mixing with newly synthesized histones would be expected (right panel). Such a scenario would be in agreement with experiments in the late 1980s involving the analysis of bulk histones without any particular focus on a specific genomic region (Prior et al. 1980; Jackson 1987, 1988; Annunziato 1990; Yamasu and Senshu 1990; Wolffe 1998; Henikoff et al. 2004). In such a scheme, parental $\mathrm{H} 3-\mathrm{H} 4$ tetramers that contain epigenetic information that do not necessarily evenly segregate onto the two daughter strands may dilute parental information that could be used in a templating mechanism. This may not necessarily be a major issue in genome regions that are organized in a repetitive manner and in which modification (such as amino-terminal histone modifications or associated proteins) of the neighbor can propagate the information. However, it is perhaps more difficult to envision how local marks spanning over a limited number of nucleosomes could be reestablished exactly in the same manner on the new DNA strands. In this context, it may be interesting to consider an alternative scenario. For example, if disruption of the tetramer can be promoted under specific circumstances, then a mixing event between parental and newly synthesized histones may occur (Fig. 5, middle panel). In this way, nucleosome core particles formed on newly synthesized DNA would inherit parental H3-H4 dimers with their encoded epigenetic information, an option that could solve the problem of inheritance of nucleosomal patterns restricted to a limited region.

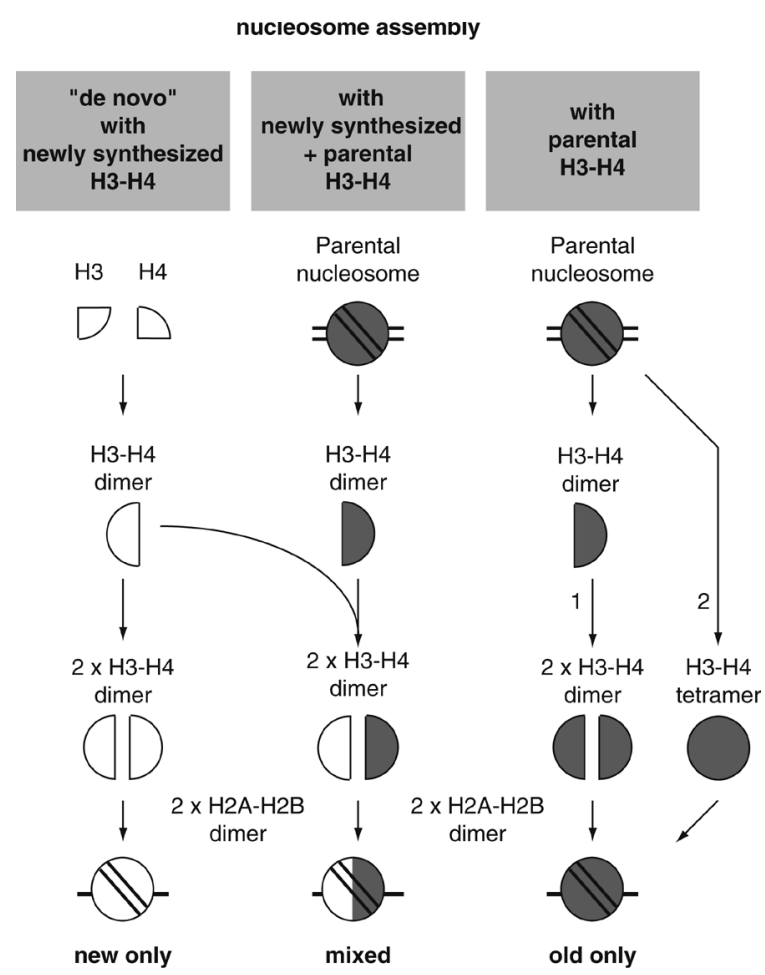

Figure 5. Dimer intermediates during nucleosome assembly. Three hypothetical and nonexclusive models of nucleosome assembly using $\mathrm{H} 3-\mathrm{H} 4$ dimer intermediates can be envisaged. Left: "De novo" assembly uses newly synthesized H3 and H4 histones to form two $\mathrm{H} 3-\mathrm{H} 4$ dimers, which, after association with two H2A-H2B dimers, leads to a nucleosome containing only new H3-H4. Middle: Assembly using a H3-H4 dimer of newly synthesized histones and a $\mathrm{H} 3-\mathrm{H} 4$ dimer recycled from a disrupted parental nucleosome. This could lead, after association with two H2A-H2B dimers, to a mixed nucleosome containing both newly synthesized and parental H3-H4. Right: Assembly using either (1) two H3-H4 dimers recycled from a transiently disrupted parental nucleosome that self-reassociate or (2) a H3-H4 tetramer stably inherited from parental nucleosome. After association with two H2A-H2B dimers, a nucleosome containing only old $\mathrm{H} 3-\mathrm{H} 4$ is formed.

This is purely hypothetical at this stage, and, of course, much work needs to be done to examine how preexisting nucleosomes can be disrupted and which machineries are capable of promoting such events associated with either replication, repair, or transcription. The stability of the tetramer entity, largely supported by many biophysical studies (D'Anna and Isenberg 1974; van Holde 1989; Karantza et al. 1996), although recently challenged (Banks and Gloss 2003), does not exclude the possibility of in vivo mechanisms to deal with the problem. The recent example of histone eviction as a mechanism underlying transcriptional activation at the $\mathrm{PHO} 5$ promoter in yeast (Boeger et al. 2003, 2004; Reinke and Horz 2003) is one illustration. Furthermore, the idea of histone exchange has been supported by recent data; in the case of variants of $\mathrm{H} 2 \mathrm{~A}$, such as $\mathrm{H} 2 \mathrm{AZ}$, the combined action of histone chaperone and remodeling factors could facilitate the reaction (Mizuguchi et al. 2004). Evidence in live cells using an elegant system to follow transcriptional ac- 
tivation has also shown appearance of H3.3 while detection of methylation on $\mathrm{H} 3 \mathrm{~K} 9$ was lost (Janicki et al. 2004). However, whether the presence of H3.3 is a cause or a consequence of transcription remains an open issue. Given that, somehow, histone exchange may be at work in the examples mentioned above, to incorportate H3.3, remodeling factors that could use ATP to disrupt preexisting histone interaction may act in combination with specific chaperones like HIRA (or CAF-1). It will thus be interesting in this respect to examine whether remaining polypeptides among the complexes containing $\mathrm{H} 3.3$ or H3. 1 correspond to known remodeling factors. The connection with transcription is particularly tempting, especially because the idea of a switch from "closed to open configuration" in chromatin is thought to occur with profound changes in nucleosomal conformation, where structural elements initially hidden become exposed. For instance, cysteine residue 110 on histone $\mathrm{H} 3$ can be chemically modified in transcribed regions (Prior et al. 1983). Our scheme summarizes possibilities that can be considered theoretically, and hopefully adequate systems will be developed to test them, taking advantage of all the recent advances in the chromatin field.

In conclusion, we found the existence of two distinct nucleosome assembly pathways that promote specific deposition of histone H3.1 or H3.3. How many more pathways remain to be identified is still an open question. The issue of dimeric histone intermediates, still provocative at this stage, is likely to stimulate future work and provides a working model that may enable our vision of nucleosome dynamics to evolve. It will be fascinating over the next few years to learn more about the many faces of nucleosome dynamics. Hopefully this will help in finding a solution to the puzzle of the inheritance of epigenetic information.

\section{ACKNOWLEDGMENTS}

We thank E. Heard and A. Loyola for critical reading. Y.N. is supported by grants from NIH (GM065939-02) and G.A. is supported by la Ligue Nationale contre le Cancer (Equipe labellisée la Ligue), Euratom (FIGH-CT1999-00010, FIGH-CT-2002-00207), Commissariat à l'Energie Atomique (LRC \#.26), Curie Program on epigenetic parameters, and NoE "Epigenome" (LSHG-CT2004-503433).

\section{REFERENCES}

Adkins M.W., Howar S.R., and Tyler J.K. 2004. Chromatin disassembly mediated by the histone chaperone Asf1 is essential for transcriptional activation of the yeast $\mathrm{PHO} 5$ and $\mathrm{PHO} 8$ genes. Mol. Cell 14: 657.

Ahmad K. and Henikoff S. 2002. The histone variant H3.3 marks active chromatin by replication-independent nucleosome assembly. Mol. Cell 9: 1191.

Aho S., Buisson M., Pajunen T., Ryoo Y.W., Giot J.F., Gruffat H., Sergeant A., and Uitto J. 2000. Ubinuclein, a novel nuclear protein interacting with cellular and viral transcription factors. J. Cell Biol. 148: 1165.

Alekseev O.M., Bencic D.C., Richardson R.T., Widgren E.E., and O'Rand M.G. 2003. Overexpression of the Linker his- tone-binding protein tNASP affects progression through the cell cycle. J. Biol. Chem. 278: 8846.

Annunziato A.T. 1990. Chromatin replication and nucleosome assembly. In Eukaryotic nucleus: Molecular biochemistry and macromolecular assemblies (ed. P.R. Strauss and S. Wilson), vol. 2, p. 687. Telford Press, Caldwell, New Jersey.

Banks D.D. and Gloss L.M. 2003. Equilibrium folding of the core histones: The H3-H4 tetramer is less stable than the H2A-H2B dimer. Biochemistry 42: 6827.

Boeger H., Griesenbeck J., Strattan J.S., and Kornberg R.D. 2003. Nucleosomes unfold completely at a transcriptionally active promoter. Mol. Cell 11: 1587.

- 2004. Removal of promoter nucleosomes by disassembly rather than sliding in vivo. Mol. Cell 14: 667.

D'Anna J.A., Jr. and Isenberg I. 1974. A histone cross-complexing pattern. Biochemistry 13: 4992.

Dignam J.D., Lebovitz R.M., and Roeder R.G. 1983. Accurate transcription initiation by RNA polymerase II in a soluble extract from isolated mammalian nuclei. Nucleic Acids Res. 11: 1475.

Felsenfeld G. and Groudine M. 2003. Controlling the double helix. Nature 421: 448.

Franklin S.G. and Zweidler A. 1977. Non-allelic variants of histones 2a, $2 \mathrm{~b}$ and 3 in mammals. Nature 266: 273.

Gaillard P.H., Martini E.M., Kaufman P.D., Stillman B., Moustacchi E., and Almouzni G. 1996. Chromatin assembly coupled to DNA repair: A new role for chromatin assembly factor I. Cell 86: 887.

Grewal S.I. and Elgin S.C. 2002. Heterochromatin: New possibilities for the inheritance of structure. Curr. Opin. Genet. Dev. 12: 178.

Heard E. 2004. Recent advances in X-chromosome inactivation. Curr. Opin. Cell Biol. 16: 247.

Henikoff S., Furuyama T., and Ahmad K. 2004. Histone variants, nucleosome assembly and epigenetic inheritance. Trends Genet. 20: 320.

Jackson V. 1987. Deposition of newly synthesized histones: New histones $\mathrm{H} 2 \mathrm{~A}$ and $\mathrm{H} 2 \mathrm{~B}$ do not deposit in the same nucleosome with new histones $\mathrm{H} 3$ and H4. Biochemistry 26: 2315.

1988. Deposition of newly synthesized histones: Hybrid nucleosomes are not tandemly arranged on daughter DNA strands. Biochemistry 27: 2109.

Jakel S., Mingot J.M., Schwarzmaier P., Hartmann E., and Gorlich D. 2002. Importins fulfill a dual function as nuclear import receptors and cytoplasmic chaperones for exposed basic domains. EMBO J. 21: 377.

Janicki S.M., Tsukamoto T., Salghetti S.E., Tansey W.P., Sachidanandam R., Prasanth K.V., Ried T., Shav-Tal Y., Bertrand E., Singer R.H., and Spector D.L. 2004. From silencing to gene expression: Real-time analysis in single cells. Cell 116: 683.

Jenuwein T. and Allis C.D. 2001. Translating the histone code. Science 293: 1074.

Karantza V., Freire E., and Moudrianakis E.N. 1996. Thermodynamic studies of the core histones: $\mathrm{pH}$ and ionic strength effects on the stability of the $(\mathrm{H} 3-\mathrm{H} 4) /(\mathrm{H} 3-\mathrm{H} 4) 2$ system. Biochemistry 35: 2037.

Koundrioukoff S., Polo S., and Almouzni G. 2004. Interplay between chromatin and cell cycle checkpoints in the context of ATR/ATM-dependent checkpoints. DNA Repair 3: 969.

Kurdistani S.K. and Grunstein M. 2003. Histone acetylation and deacetylation in yeast. Nat. Rev. Mol. Cell Biol. 4: 276.

Lamour V., Lecluse Y., Desmaze C., Spector M., Bodescot M., Aurias A., Osley M.A., and Lipinski M. 1995. A human homolog of the $S$. cerevisiae HIR1 and HIR2 transcriptional repressors cloned from the DiGeorge syndrome critical region. Hum. Mol. Genet. 4: 791.

Laskey R.A., Mills A.D., and Morris N.R. 1977. Assembly of SV40 chromatin in a cell-free system from Xenopus eggs. Cell 10: 237.

Loyola A. and Almouzni G. 2004. Histone chaperones, a supporting role in the limelight. Biochim. Biophys. Acta 15: 1.

Maison C. and Almouzni G. 2004. HP1 and the dynamics of heterochromatin maintenance. Nat. Rev. Mol. Cell Biol. 5: 296.

McKittrick E., Gafken P.R., Ahmad K., and Henikoff S. 2004. 
Histone H3.3 is enriched in covalent modifications associated with active chromatin. Proc. Natl. Acad. Sci. 101: 1525.

Mello J.A. and Almouzni G. 2001. The ins and outs of nucleosome assembly. Curr. Opin. Genet. Dev. 11: 136.

Mello J.A., Sillje H.H., Roche D.M., Kirschner D.B., Nigg E.A., and Almouzni G. 2002. Human Asf1 and CAF-1 interact and synergize in a repair-coupled nucleosome assembly pathway. EMBO Rep. 3: 329.

Mizuguchi G., Shen X., Landry J., Wu W.H., Sen S., and Wu C. 2004. ATP-driven exchange of histone $\mathrm{H} 2 \mathrm{AZ}$ variant catalyzed by SWR1 chromatin remodeling complex. Science 303: 343.

Munakata T., Adachi N., Yokoyama N., Kuzuhara T., and Horikoshi M. 2000. A human homologue of yeast anti-silencing factor has histone chaperone activity. Genes Cells 5: 221.

Nakatani Y. and Ogryzko V. 2003. Immunoaffinity purification of mammalian protein complexes. Methods Enzymol. 370: 430.

Nelson D.M., Ye X., Hall C., Santos H., Ma T., Kao G.D., Yen T.J., Harper J.W., and Adams P.D. 2002. Coupling of DNA synthesis and histone synthesis in S phase independent of cyclin/cdk2 activity. Mol. Cell. Biol. 22: 7459.

Prior C.P., Cantor C.R., Johnson E.M., and Allfrey V.G. 1980. Incorporation of exogenous pyrene-labeled histone into Physarum chromatin: A system for studying changes in nucleosomes assembled in vivo. Cell 20: 597.

Prior C.P., Cantor C.R., Johnson E.M., Littau V.C., and Allfrey V.G. 1983. Reversible changes in nucleosome structure and histone $\mathrm{H} 3$ accessibility in transcriptionally active and inactive states of rDNA chromatin. Cell 34: 1033.

Quivy J.P., Roche D., Kirschner D., Tagami H., Nakatani Y., and Almouzni G. 2004. A CAF-1 dependent pool of HP1 during heterochromatin duplication. $E M B O J$. 12: 12.

Ray-Gallet D. and Almouzni G. 2004. DNA synthesis-dependent and -independent chromatin assembly pathways in Xenopus egg extracts. Methods Enzymol. 375: 117.

Ray-Gallet D., Quivy J.P., Scamps C., Martini E.M., Lipinski M., and Almouzni G. 2002. HIRA is critical for a nucleosome assembly pathway independent of DNA synthesis. Mol. Cell 9: 1091 .

Reinke H. and Horz W. 2003. Histones are first hyperacetylated and then lose contact with the activated PHO5 promoter. Mol. Cell 11: 1599.

Richardson R.T., Batova I.N., Widgren E.E., Zheng L.X., Whitfield M., Marzluff W.F., and O'Rand M.G. 2000. Characteri- zation of the histone H1-binding protein, NASP, as a cell cycle-regulated somatic protein. J. Biol. Chem. 275: 30378.

Ridgway P. and Almouzni G. 2000. CAF-1 and the inheritance of chromatin states: At the crossroads of DNA replication and repair. J. Cell Sci. 113: 2647.

Sherwood P.W., Tsang S.V., and Osley M.A. 1993. Characterization of HIR1 and HIR2, two genes required for regulation of histone gene transcription in Saccharomyces cerevisiae. Mol. Cell. Biol. 13: 28.

Sillje H.H. and Nigg E.A. 2001. Identification of human Asf1 chromatin assembly factors as substrates of Tousled-like kinases. Curr. Biol. 11: 1068.

Smith S. and Stillman B. 1989. Purification and characterization of CAF-I, a human cell factor required for chromatin assembly during DNA replication in vitro. Cell 58: 15.

Sun L., Youn H.D., Loh C., Stolow M., He W., and Liu J.O. 1998. Cabin 1, a negative regulator for calcineurin signaling in T lymphocytes. Immunity 8: 703.

Tagami H., Ray-Gallet D., Almouzni G., and Nakatani Y. 2004. Histone H3.1 and H3.3 complexes mediate nucleosome assembly pathways dependent or independent of DNA synthesis. Cell 116: 51.

Turner B.M. 2002. Cellular memory and the histone code. Cell 111: 285.

Tyler J.K., Collins K.A., Prasad-Sinha J., Amiott E., Bulger M., Harte P.J., Kobayashi R., and Kadonaga J.T. 2001. Interaction between the Drosophila CAF-1 and ASF1 chromatin assembly factors. Mol. Cell. Biol. 21: 6574 .

van Holde K. 1989. Chromatin. Springer-Verlag, New York.

Verreault A. 2000. De novo nucleosome assembly: New pieces in an old puzzle. Genes Dev. 14: 1430.

Waterborg J.H. 1990. Sequence analysis of acetylation and methylation in two histone $\mathrm{H} 3$ variants of alfalfa. J. Biol. Chem. 265: 17157.

Watson J.D. and Crick F.H.C. 1953. Molecular structure of nucleic acids. Nature 171: 737.

Wolffe A. 1998. Chromatin: Structure and function, 3rd edition. Academic Press, San Diego, California.

Wu R.S., Tsai S., and Bonner W.M. 1982. Patterns of histone variant synthesis can distinguish G0 from G1 cells. Cell 31: 367.

Yamasu K. and Senshu T. 1990. Conservative segregation of tetrameric units of $\mathrm{H} 3$ and $\mathrm{H} 4$ histones during nucleosome replication. J. Biochem. 107: 15. 


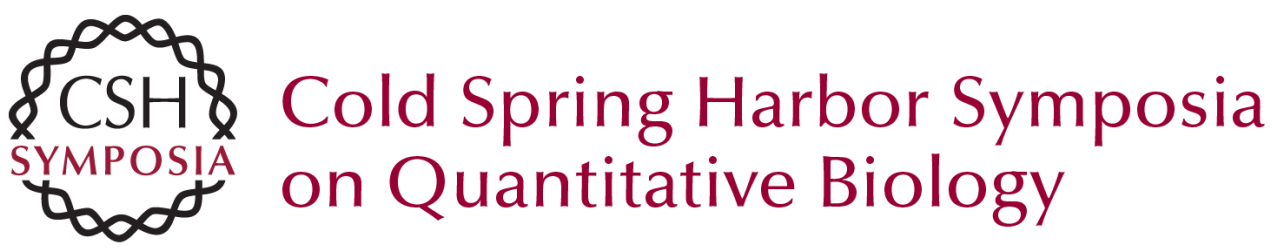

\section{Two Distinct Nucleosome Assembly Pathways: Dependent or Independent of DNA Synthesis Promoted by Histone H3.1 and H3.3 Complexes}

Y. NAKATANI, D. RAY-GALLET, J.-P. QUIVY, et al.

Cold Spring Harb Symp Quant Biol 2004 69: 273-280

Access the most recent version at doi:10.1101/sqb.2004.69.273

References This article cites 55 articles, 14 of which can be accessed free at: http://symposium.cshlp.org/content/69/273.full.html\#ref-list-1

\section{License}

Email Alerting Receive free email alerts when new articles cite this article - sign up in Service the box at the top right corner of the article or click here. 